\title{
Commentary \\ Leukocyte phenotyping to stratify septic shock patients
} Jonathan E McDunn and Richard S Hotchkiss

Department of Anesthesiology, Washington University School of Medicine, 660 South Euclid Avenue, Saint Louis, MI 63110, USA

Corresponding author: Richard S Hotchkiss, hotchkir@anest.wustl.edu

See related research by Monserrat et al., http://ccforum.com/content/13/1/R26

Published: 3 April 2009

This article is online at http://ccforum.com/content/13/2/127

(c) 2009 BioMed Central Ltd

Critical Care 2009, 13:127 (doi:10.1186/cc7748)

C2009 BioMed Central Ltd

\begin{abstract}
In a recent study conducted in a cohort of 52 septic patients, Monserrat and coworkers found that profound failure of peripheral T cells to convert from a naïve phenotype to an activated phenotype has positive predictive value in identifying patients who do not recover. These data support the hypothesis that failure of the innate immune system to engage the T-cell compartment contributes to sepsis mortality and provides motivation for the development and clinical evaluation of immunostimulatory therapies for patients with sepsis.
\end{abstract}

Clinical scoring systems stratify patients on a population basis, but they do not have sufficient sensitivity and specificity to answer fundamental questions about individuals. For instance, is the patient infected, responding to therapy, or likely to recover? In this issue, Monserrat and coworkers [1] report their examination of surface marker expression on peripheral T cells over a 28-day period (intensive care unit [ICU] admission through follow up) in 52 patients with septic shock and 36 age-matched and sex-matched healthy control individuals. Consistent with previous reports, this cohort of septic patients had significantly depressed T-cell numbers on ICU admission that persisted for weeks $[2,3]$. Monserrat and coworkers retrospectively classified patients as either survivors or nonsurvivors and showed that, with the exception of injury severity assessments (Acute Physiology and Chronic Health Evaluation II, Multiple Organ Dysfunction Score, Sepsis-related Organ Failure Assessment), these two groups of patients were similar on enrollment.

Although all septic patients had similarly depressed numbers of $\mathrm{CD}^{+}$and $\mathrm{CD8}^{+}$lymphocytes, serial profiling of the absolute abundance of naïve lymphocytes with different phenotypic markers revealed significant differences between survivors and nonsurvivors. The number of cells expressing surface markers of the naïve phenotype was significantly higher in nonsurvivors than in survivors.
Other biomarker studies of ICU populations have examined additional patient populations (critically ill nonseptic patients, and patients with sepsis and severe sepsis) as well as healthy control individuals and patients with septic shock. The comparison between septic shock and health reveals the most profound differences, and it is easiest to power a study with just those two groups. However, it would be valuable to know whether Monserrat and coworkers' leukocyte phenotype has utility outside the diagnosis of septic shock. No standard critical care protocol was followed for this study, and it is possible that current patient management regimens do not effectively treat the nonsurvivor patient cohort.

These findings add to the body of leukocyte phenotyping studies that aim to differentiate patients with syndromic diagnoses that are common in the ICU setting [2-9]. Results from single-center studies have illustrated that there are multiple leukocyte phenotypes with potential clinical utility for either diagnosis or prognostication. Monserrat and coworkers [1] found that the best receiver operator characteristic (ROC) curve based on a single variable was obtained with data obtained from ICU admission, and a cut-off of 136 $\mathrm{CD}^{+}{ }^{+} \mathrm{CD}_{28}{ }^{+}$T-cells per microliter exhibited $70 \%$ sensitivity and 100\% specificity for predicting death (area under the curve $[A U C]=0.84$ ).

In earlier work, Saenz and coworkers [4] identified a monocyte phenotype (total number of monocytes per microliter $[=445]$ + percentage of monocytes that were CD13/HLA-DR positive [32\%]) that distinguished survivors from nonsurvivors in patients with severe sepsis (AUC $=0.918$ ). By combining two informative variables, those investigators extracted additional power from their data. Tschaikowsky and coworkers [5] have already shown that lymphocyte and monocyte markers exhibit concordant changes in a cohort of patients with severe sepsis. It would be valuable to know

$\mathrm{AUC}=$ area under the curve; $I \mathrm{CU}=$ intensive care $\mathrm{unit} ; \mathrm{ROC}=$ receiver operating characteristic. 
whether combining the positive predictors from the study by Monserrat and coworkers [1], or whether combining lymphocyte and monocyte phenotyping in the same study, would improve the ROC.

This study raises numerous questions. If survivors are responding to therapy and recovering, then why was the ROC performance best at ICU admission? Is it because of loss of individuals from the nonsurvivor cohort, reducing statistical power at subsequent time points, or is the immune compartment in nonsurvivors responding to therapy even though these patients die? Additional demographic information would be valuable to assist in interpreting these data, especially regarding existing co-morbid conditions and the admitting diagnosis. Based on the results of interventional trials, it is clear that the type of patient and ICU can influence the efficacy or utility of a test or intervention (for example, intensive insulin therapy has different effects on outcome depending on whether it is instituted in a surgical or medical ICU setting $[10,11])$. If leukocyte phenotyping conclusively identifies a group of patients who cannot be rescued by current care, then how should physicians use this information?

From a mechanistic standpoint, these data add support to the hypothesis that failure of the innate immune system to engage the T-cell compartment plays a significant role in sepsis mortality [12] and suggest that immune-supportive or immunostimulatory therapies may be of value in the management of the septic patient [13]. Further development of a leukocyte phenotyping-based test for ICU patient stratification beyond clinical scoring could aid in both diagnosis and prognosis and, if validated in a prospective patient cohort, could be incorporated into both clinical practice and clinical trial design.

\section{Competing interests}

The authors declare that they have no competing interests.

\section{References}

1. Monserrat J, De Pablo R, Reyes E, Diaz D, Barcenilla H, Zapata MR, De La Hera A, Prieto A, Alvarez-Mon M: Clinical relevance of the severe abnormalities of the T cell compartment in septic shock patients. Crit Care 2009, 13:R26.

2. Lin RY, Astiz ME, Saxon JC, Rackow EC: Altered leukocyte immunophenotypes in septic shock. Studies of HLA-DR, CD11b, CD14, and IL-2R expression. Chest 1993, 104:847853.

3. Cheadle WG, Pemberton RM, Robinson D, Livingston DH, Rodriguez JL, Polk HC Jr: Lymphocyte subset responses to trauma and sepsis. J Trauma 1993, 35:844-849.

4. Sáenz JJ, Izura JJ, Manrique A, Sala F, Gaminde I: Early prognosis in severe sepsis via analyzing the monocyte immunophenotype. Intensive Care Med 2001, 27:970-977.

5. Tschaikowsky K, Hedwig-Geissing M, Schiele A, Bremer F, Schywalsky M, Schuettler J: Coincidence of pro- and anti-inflammatory responses in the early phase of severe sepsis: longitudinal study of mononuclear histocompatibility leukocyte antigen-DR expression, procalcitonin, C-reactive protein, and changes in T-cell subsets in septic and postoperative patients. Crit Care Med 2002, 30:1015-1023.

6. Holub M, Klucková Z, Helcl M, Prihodov J, Rokyta R, Beran O: Lymphocyte subset numbers depend on the bacterial origin of sepsis. Clin Microbiol Infect 2003, 9:202-211.
7. Roth G, Moser B, Krenn C, Brunner M, Haisjackl M, Almer G, Gerlitz S, Wolner E, Boltz-Nitulescu G, Ankersmit HJ: Susceptibility to programmed cell death in T-lymphocytes from septic patients: a mechanism for lymphopenia and Th2 predominance. Biochem Biophys Res Commun 2003, 308:840-846.

8. Faivre V, Lukaszewicz AC, Alves A, Charron D, Payen D, Haziot A: Accelerated in vitro differentiation of blood monocytes into dendritic cells in human sepsis. Clin Exp Immunol 2007, 147: 426-439.

9. Schwulst SJ, Muenzer JT, Chang KC, Brahmbhatt TS, Coopersmith CM, Hotchkiss RS: Lymphocyte phenotyping to distinguish septic from nonseptic critical illness. J Am Coll Surg 2008, 206:335-342.

10. van den Berghe $G$, Wouters $P$, Weekers F, Verwaest $C$, Bruyninckx F, Schetz M, Vlasselaers D, Ferdinande P, Lauwers P, BouilIon R: Intensive insulin therapy in critically ill patients. $N$ Engl J Med 2001, 345:1359-1367.

11. van den Berghe G, Wilmer A, Hermans G, Meersseman W, Wouters PJ, Milants I, Van Wigngaerden E, Bobbaers H, Bouillon $\mathrm{R}$ : Intensive insulin therapy in the medical ICU. $N$ Engl J Med 2006, 354:449-461.

12. Marshall JC, Charbonney E, Gonzalez PD: The immune system in critical illness. Clin Chest Med 2008, 29:605-616.

13. Lyn-Kew K, Standiford TJ: Immunosuppression in sepsis. Curr Pharm Des 2008, 14:1870-1881. 